\title{
Large-Scale Analysis of Bioactive Ligand Conformational Strain Energy Using ab Initio Calculation
}

Jiahui Tong ${ }^{\dagger, \ddagger, \S, \|}$, Suwen Zhao ${ }^{*, \dagger, \ddagger}$

†iHuman Institute, ShanghaiTech University, 393 Middle Huaxia Road, Shanghai, 201210, China

${ }^{\ddagger}$ School of Life Science and Technology, ShanghaiTech University, 393 Middle Huaxia Road, Shanghai, 201210, China

§University of Chinese Academy of Sciences, No. 19A, Yuquan Road, Beijing, 100049, China

"Shanghai Institute of Nutrition and Health, Chinese Academy of Sciences, 320 Yueyang Road, Shanghai, 200031, China

*Corresponding to: S.Z.: zhaosw@shanghaitech.edu.cn. 
Table S1. Summary of previous calculations of strain energies.

\begin{tabular}{|c|c|c|c|c|}
\hline Year & Authors & Dataset & Calculation Level & Results \\
\hline \multicolumn{5}{|c|}{ Direct calculations of ligand strain energies } \\
\hline 1995 & Nicklaus et al. ${ }^{2}$ & $\begin{array}{l}\text { Conformations from CSD and PDB of } 27 \\
\text { flexible ligands }\end{array}$ & CHARMm force field & Average ligand conformational strain energy: $15.9 \mathrm{kcal} / \mathrm{mol}$ \\
\hline 1998 & Boström et al. ${ }^{4}$ & 33 ligand bound conformations from PDB & MM3 and AMBER force field & $\begin{array}{l}\text { Ligand conformational strain energy should not less than } 3 \\
\mathrm{kcal} / \mathrm{mol} \text { ( } 9 \text { outlier in their study) }\end{array}$ \\
\hline 2004 & Perola et al. ${ }^{6}$ & $\begin{array}{l}150 \text { ligand bound conformations from PDB and } \\
\text { Vertex structure collection }\end{array}$ & MMFF and OPLS-AA & $\begin{array}{l}60 \% \text { of the ligands with strain energy lower than } 5 \mathrm{kcal} / \mathrm{mol} \\
\text { and at least } 10 \% \text { of them over } 9 \mathrm{kcal} / \mathrm{mol}\end{array}$ \\
\hline 2012 & Sitzmann et al. ${ }^{11}$ & 415 ligand bound conformations from PDB & $\begin{array}{c}\text { B3LYP/6-311++G(3df,2p)//B3LYP/6- } \\
\text { 31(d) }\end{array}$ & $\begin{array}{l}\text { Ligand conformational strain energy occurred in a range of } 0 \\
\text { to } \sim 25 \mathrm{kcal} / \mathrm{mol}\end{array}$ \\
\hline 2014 & Borbulevych et al. ${ }^{12}$ & 50 ligand bound conformations from PDB & AM1 and $\mathrm{HF} / 6-311+\mathrm{G}^{* *}$ & Average ligand conformational strain energy: $24.60 \mathrm{kcal} / \mathrm{mol}$ \\
\hline 2018 & Borbulevych et al. ${ }^{13}$ & $\begin{array}{l}\text { Ligand conformations from } 80 \text { Astex PDB } \\
\text { structures }\end{array}$ & PM6 and AMBER MM force field & Average ligand conformational strain energy: $9.95 \mathrm{kcal} / \mathrm{mol}$ \\
\hline \multicolumn{5}{|c|}{ Calculations of torsion strain energies } \\
\hline 1996 & Allen et al. ${ }^{14}$ & $\begin{array}{l}12 \text { molecular substructures (compared to } \\
\text { structures in CSD) }\end{array}$ & $\begin{array}{c}\mathrm{HF} / 6-31 \mathrm{G} * / / \mathrm{HF} / 3-21 \mathrm{G} \\
\mathrm{HF} / 6-31 \mathrm{G} * / / \mathrm{HF} / \mathrm{STO}-3 \mathrm{G}\end{array}$ & $\begin{array}{l}\text { Only } 3 \% \text { of the examples of the corresponding substructures in } \\
\text { the CSD with torsion strain energy over } 1 \mathrm{kcal} / \mathrm{mol}\end{array}$ \\
\hline 2007 & Hao et al. ${ }^{16}$ & $\begin{array}{l}21 \text { common torsion motifs of ligands (compared } \\
\text { to structures in PDB) }\end{array}$ & B3LYP/6-311++G** & $\begin{array}{l}\text { The average strain energy of these torsion motifs due to } \\
\text { binding to the protein is } \sim 0.6 \mathrm{kcal} / \mathrm{mol} \text {. }\end{array}$ \\
\hline 2019 & Brajesh et al. ${ }^{17}$ & $\begin{array}{l}\text { 30,009 unique molecular fragments (compared } \\
\text { to structures in both CSD and PDB) }\end{array}$ & $\begin{array}{c}\text { B3LYP/6-31G**(6-31+G** for sulfur- } \\
\text { containing molecules)//B3LYP/6- } \\
\text { 31G*(6-31+G* for sulfur-containing } \\
\text { molecules })\end{array}$ & $\begin{array}{l}\text { Torsion strain energy cutoff: } 2.5 \mathrm{kcal} / \mathrm{mol} \text { for global strain and } \\
1.5 \mathrm{kcal} / \mathrm{mol} \text { for local strain. }\end{array}$ \\
\hline
\end{tabular}




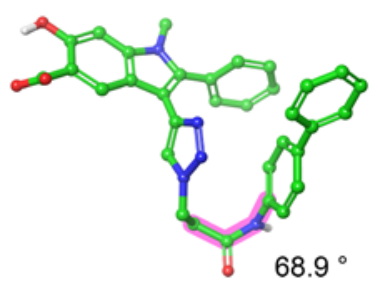

PDB: 305X Ligand: JZG

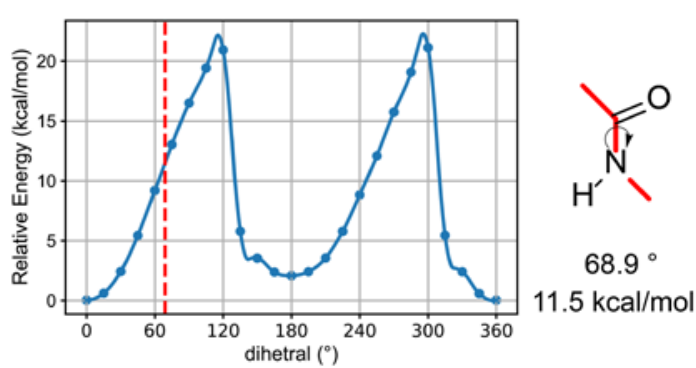

b

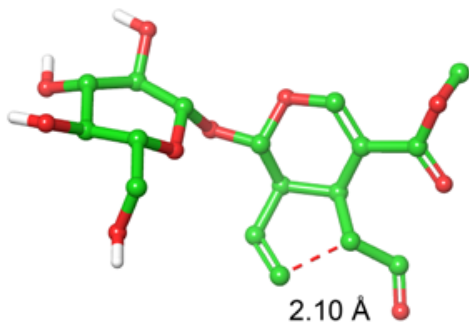

PDB: 2IGT Ligand: SAM

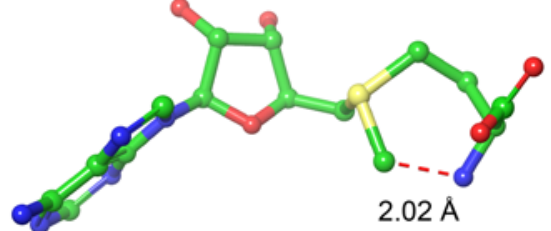

PDB: 3U5Y Ligand: SCG

Figure S1. Examples of ligand bound conformations filtered by the geometry error check. (a) An example (PDB ID: $305 \mathrm{X}^{62}$ ) in which $\mathrm{sp}^{2}$ atoms are not on a plane. Torsion energy scan of peptide bond shows very high strain energy of current dihedral angle in ligand bound conformation. (b) Two examples (PDB ID: 2IGT, 3U5Y ${ }^{63}$ ) in which there are steric clashes between heavy atoms. 


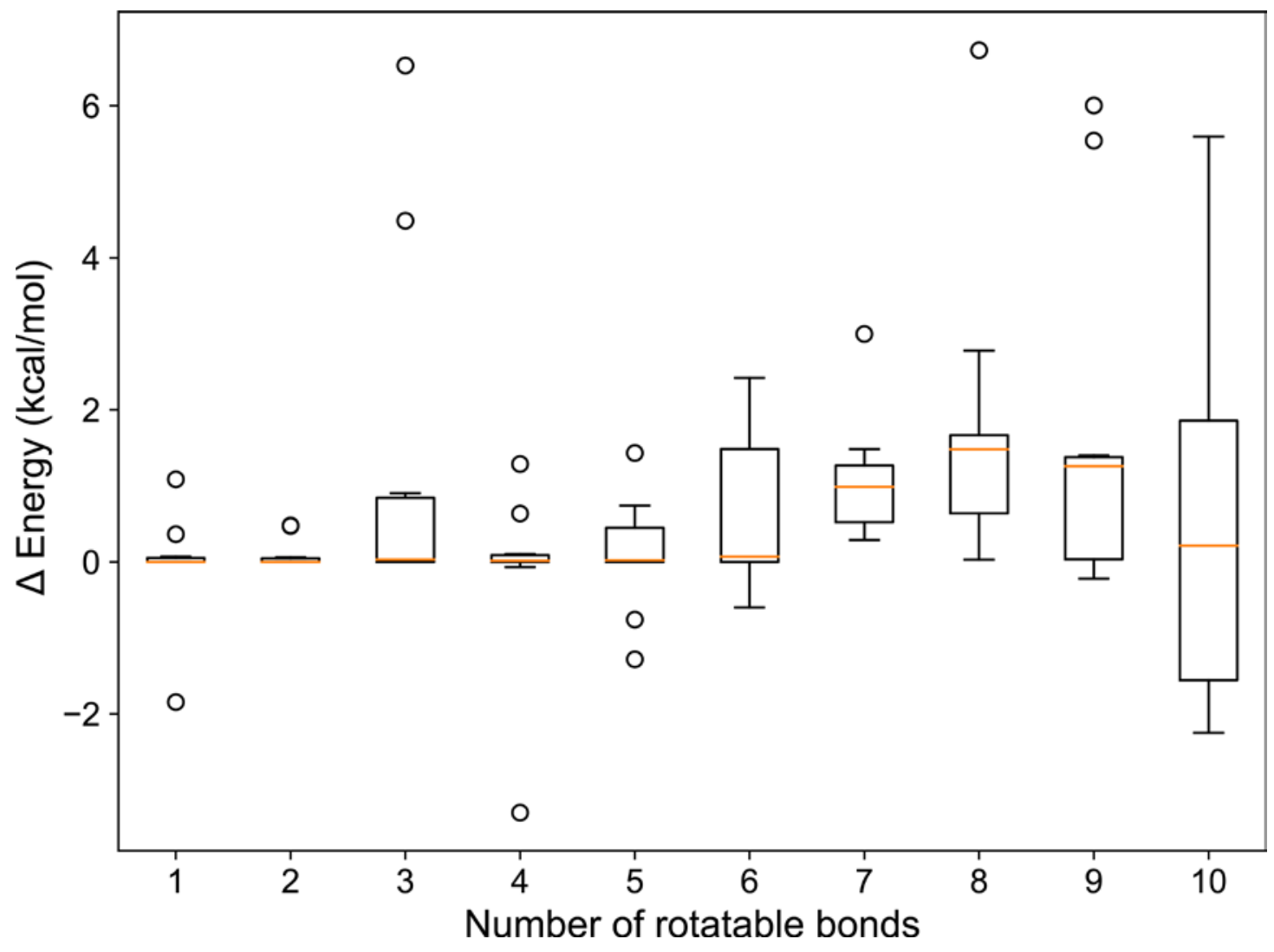

Figure S2. Distribution of the global minimum energy difference $\left(\Delta\right.$ energy $=\mathrm{E}_{\text {glom(old })}$ - Eglom(new) between old and new sampling protocols. 


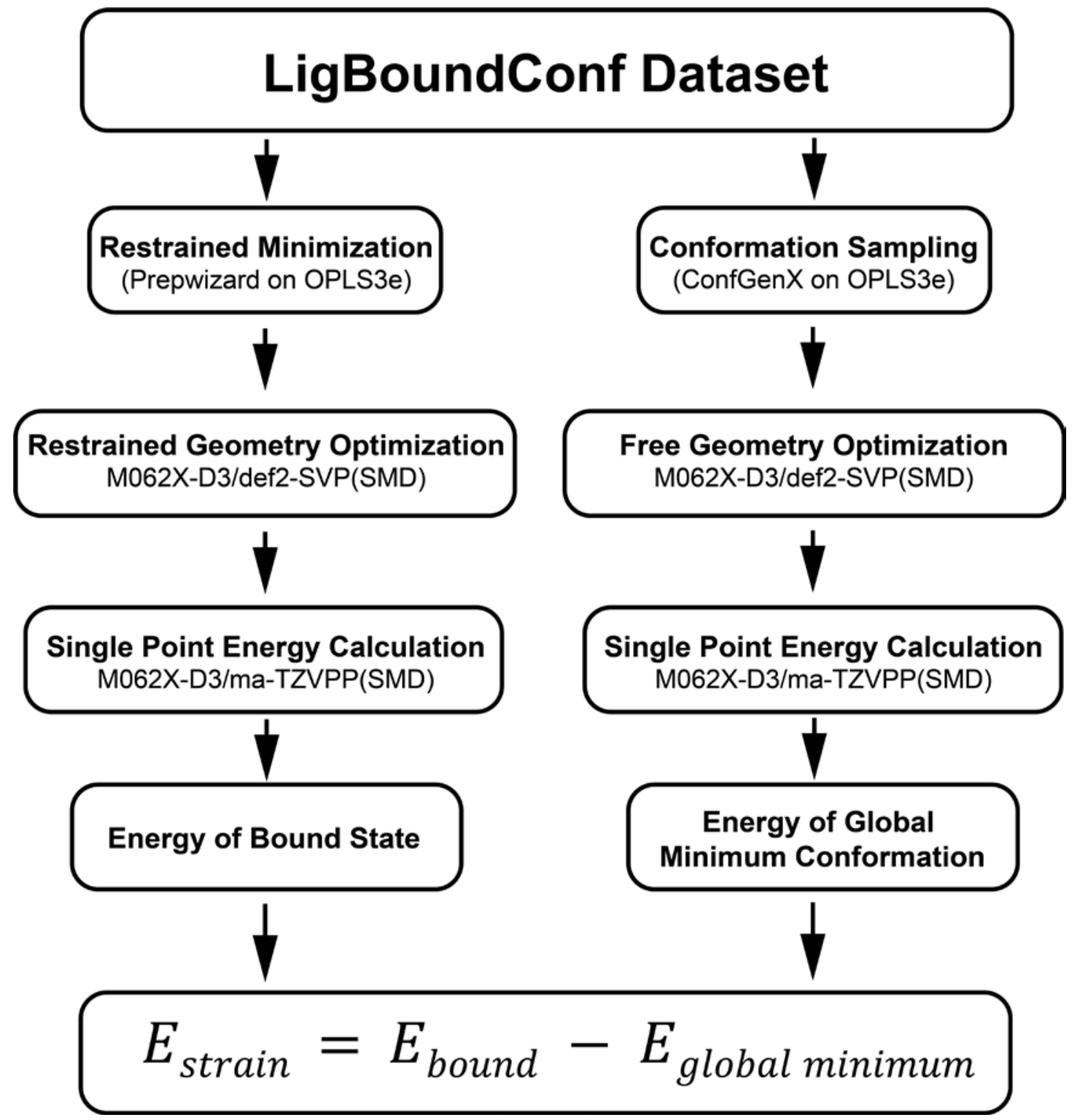

Figure S3. Workflow of the ligand conformation process and ligand strain energy calculation. 


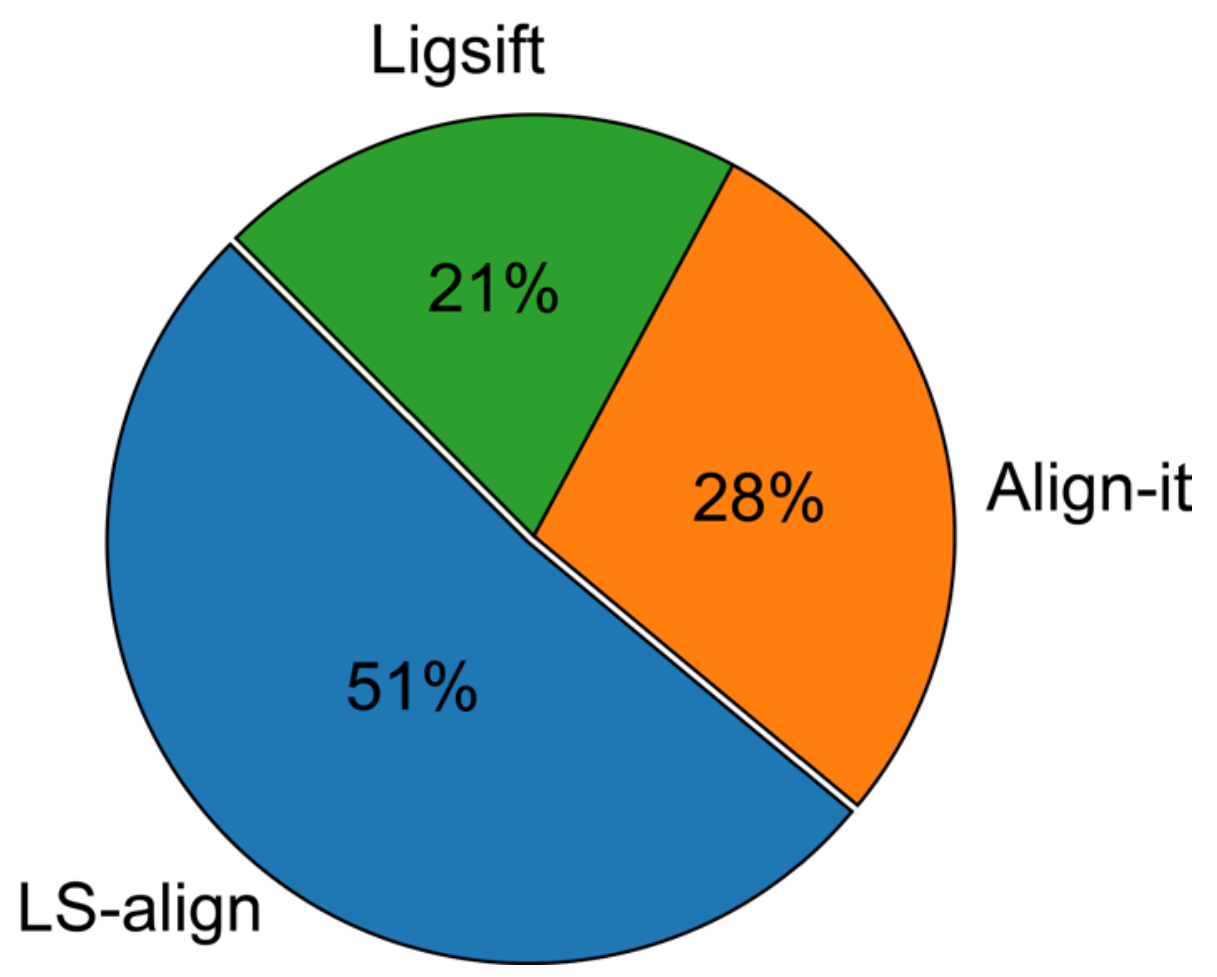

Figure S4. Contributions of alignment tools to the final alignment results. 


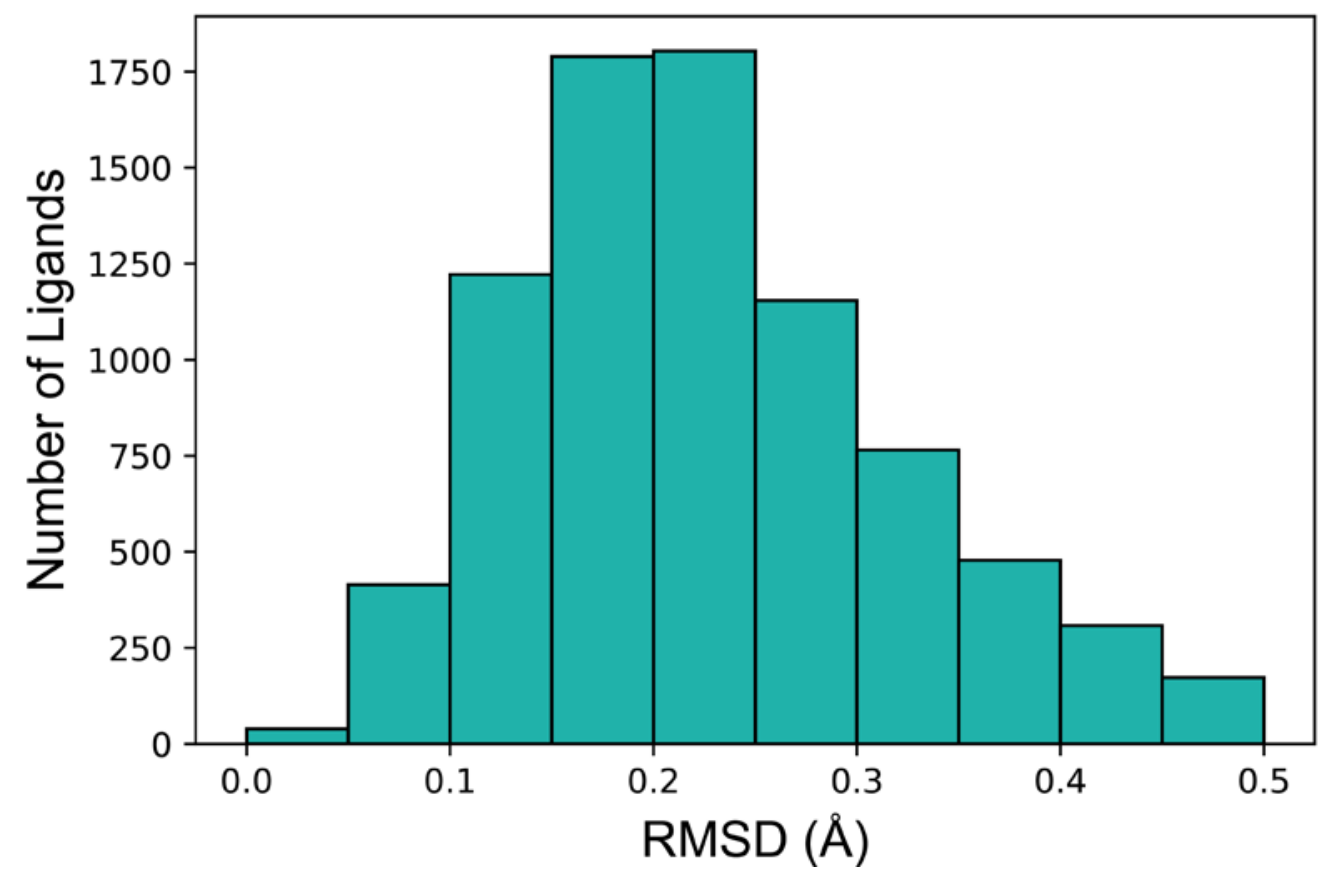

Figure S5. Ligand RMSD distribution after the pre-relaxation step. 

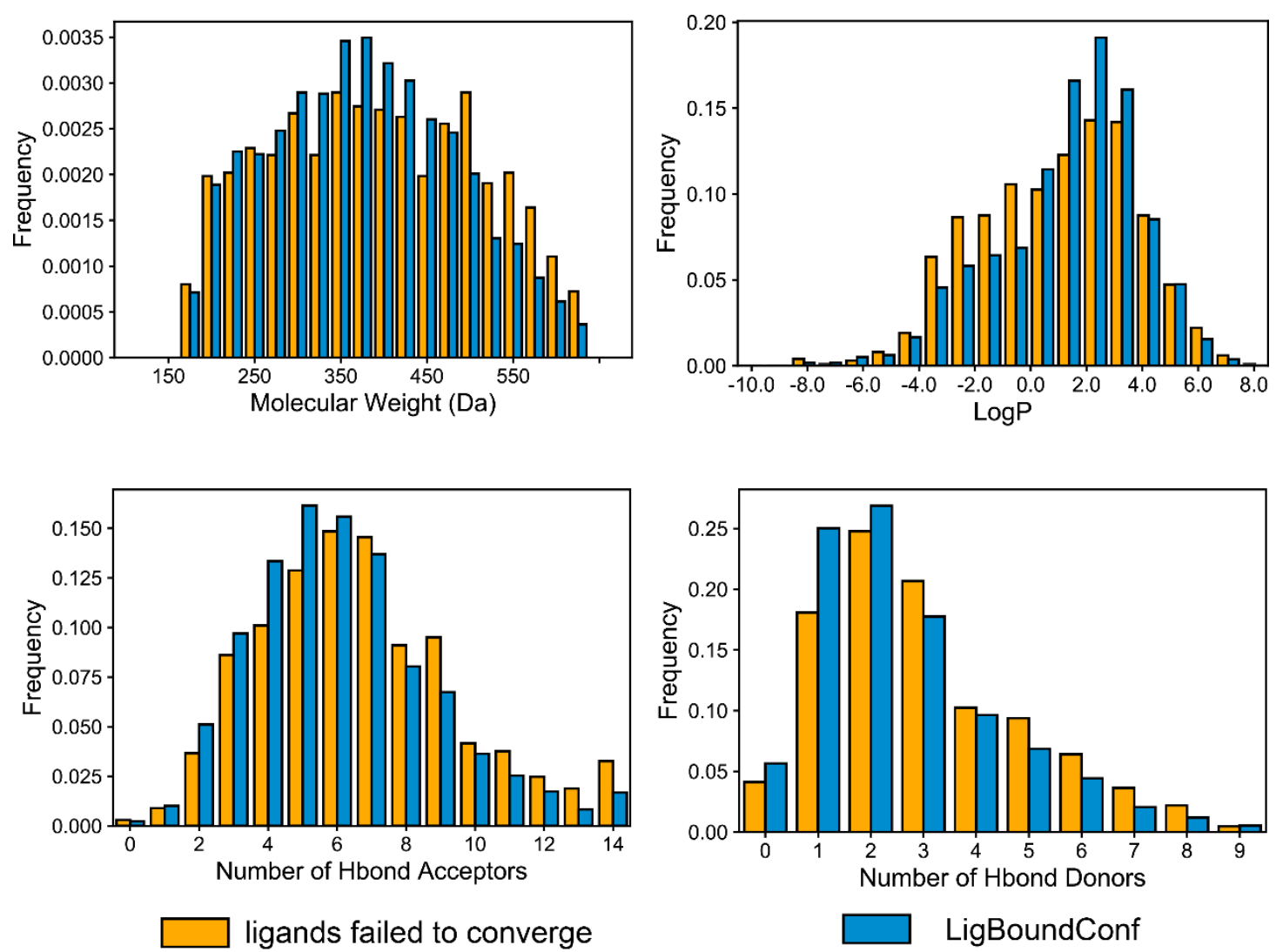

Figure S6. Comparison of physiochemical properties of ligands failed to converge and all the ligands in LigBoundConf. 


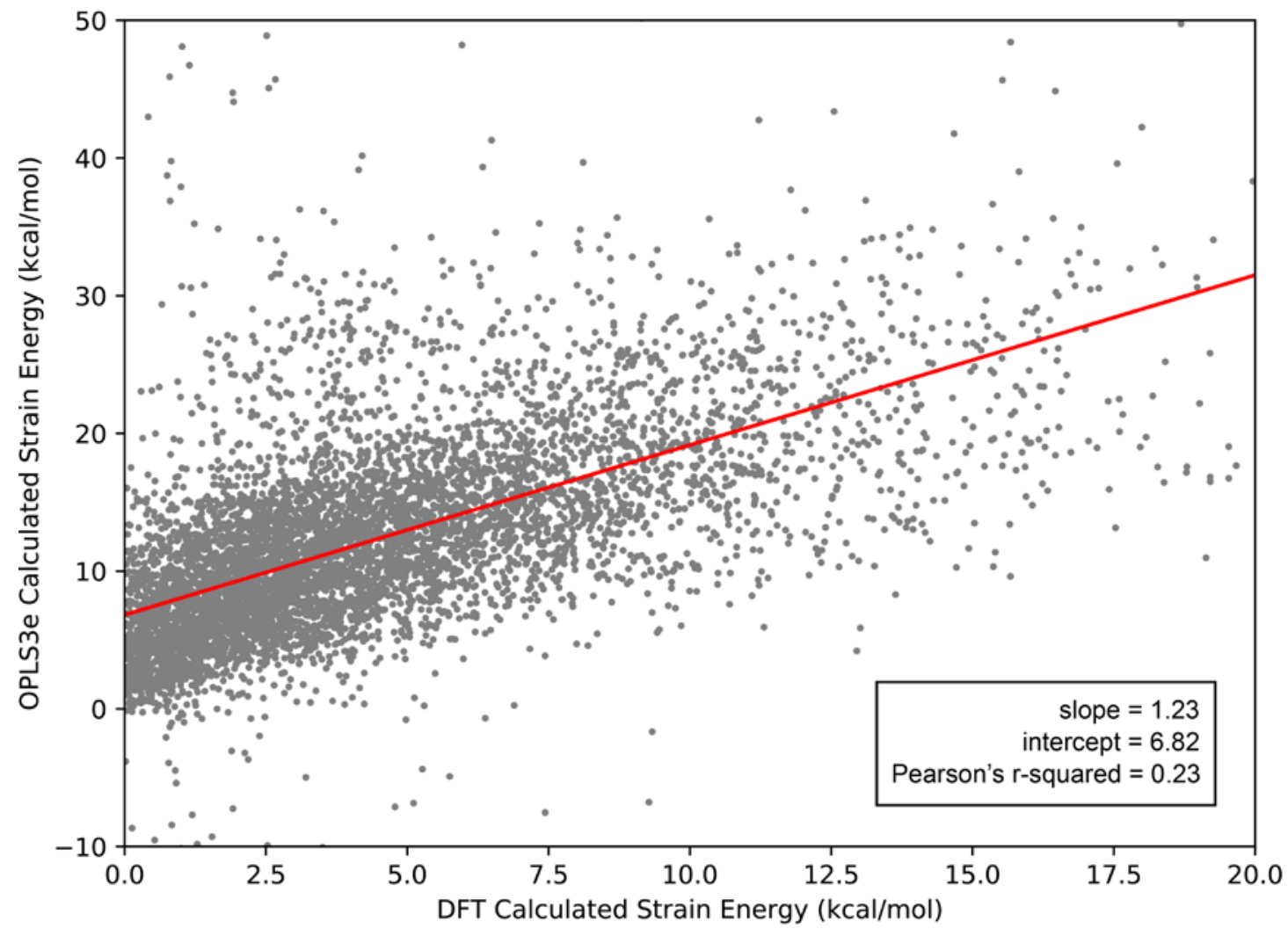

Figure S7. Comparison between strain energy obtained from DFT calculation and calculations based on OPLS3e. 20 and $50 \mathrm{kcal} / \mathrm{mol}$ cutoffs were used for DFT calculated strain energy and OPLS3e calculated strain energy, respectively, which excludes 94 outliers ( 1\%) out of the coordinate ranges. 


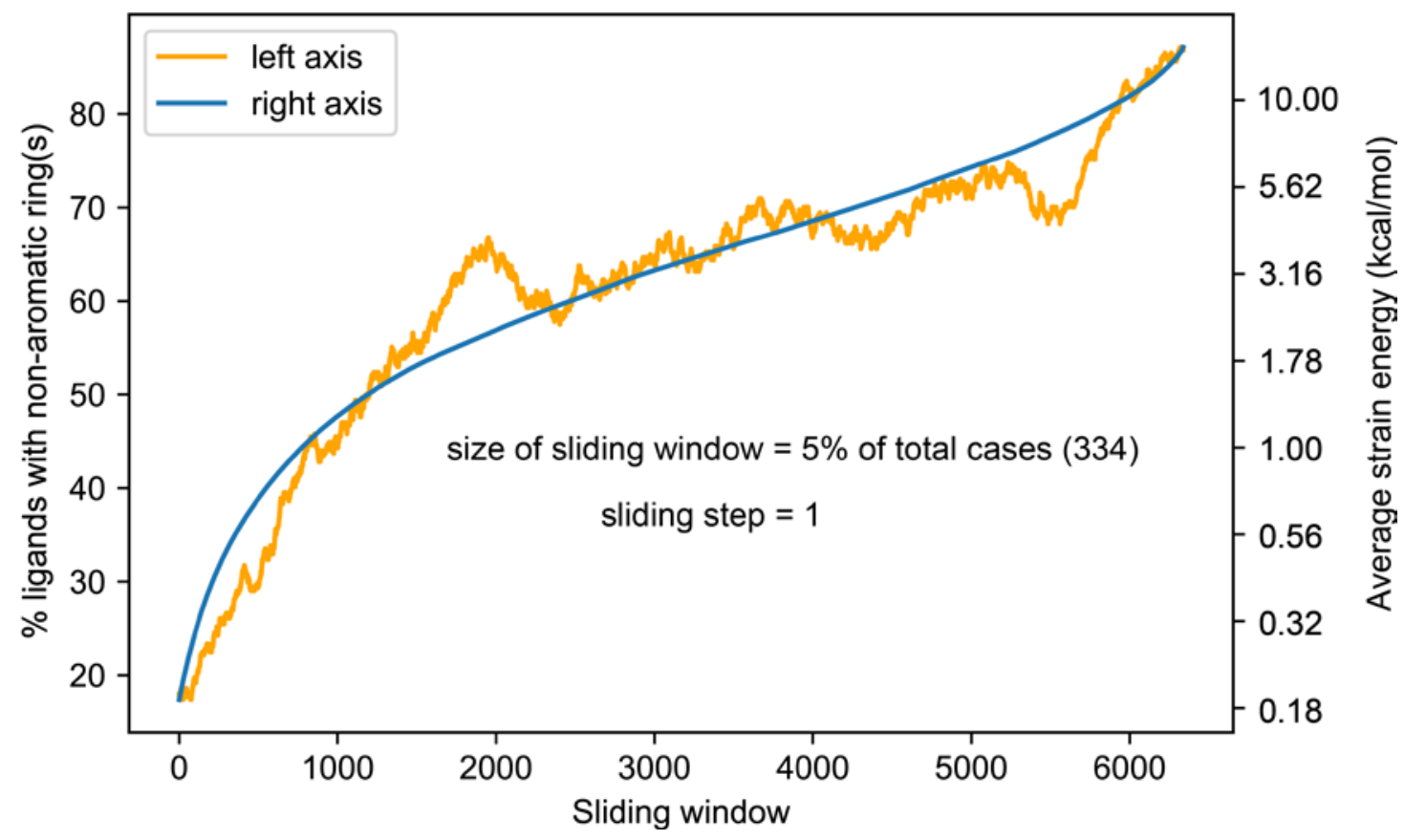

Figure S8. Sliding windows analysis on relationship between LCSE and ratio of ligands with non-aromatic ring(s). Window slides on 6,672 successfully calculated cases ranked by strain energy. Window size is 334 (5\% of 6,672) and sliding step is 1 . Each point in orange line represents ratio of ligands with non-aromatic ring(s) of cases in sliding window. Each point in blue line represents average strain energy of cases in sliding window. 

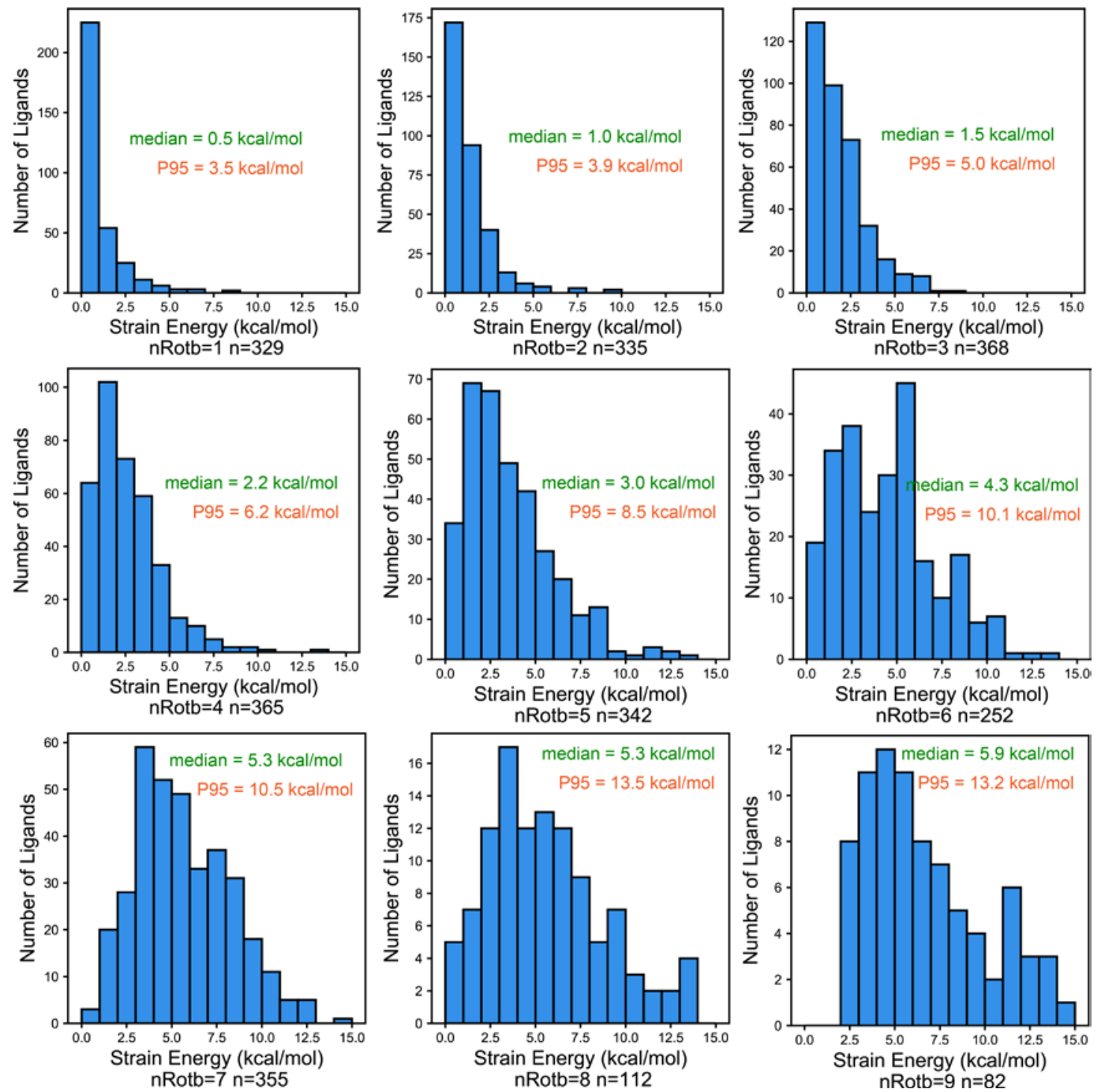

Figure S9. Distribution profiles of strain energies for ligands with different numbers of rotatable bonds, which have no non-aromatic ring(s). Median is the median number of strain energies, and P95 is the strain energy below which 95\% of LCSEs fall. 


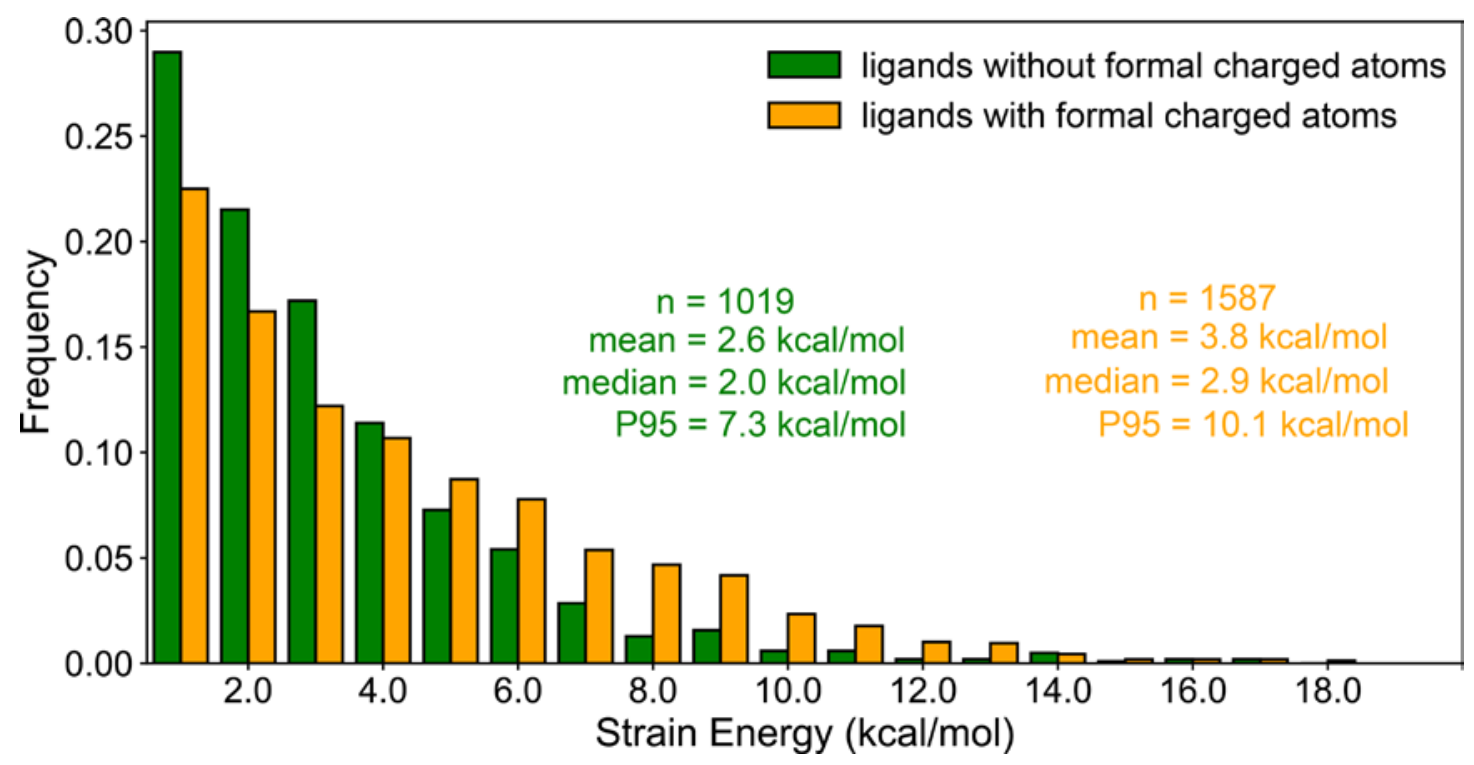

Figure S10. Comparison of strain energy distribution between ligands with and without formal charged atoms, which have no non-aromatic ring(s). 

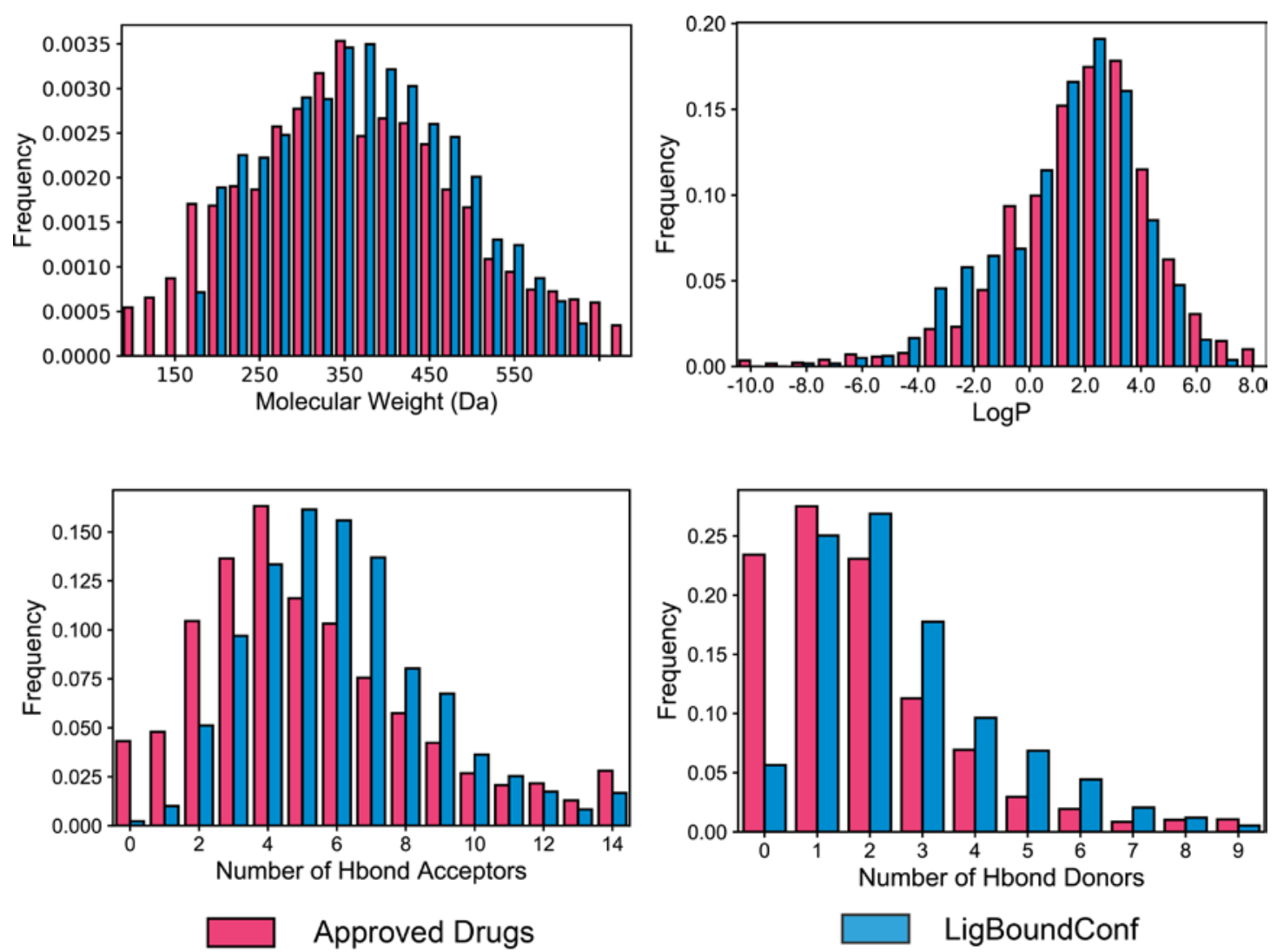

Figure S11. Distributions of ligand physicochemical properties calculated for approved drugs obtained from DrugBank and ligands in LigBoundConf dataset. 

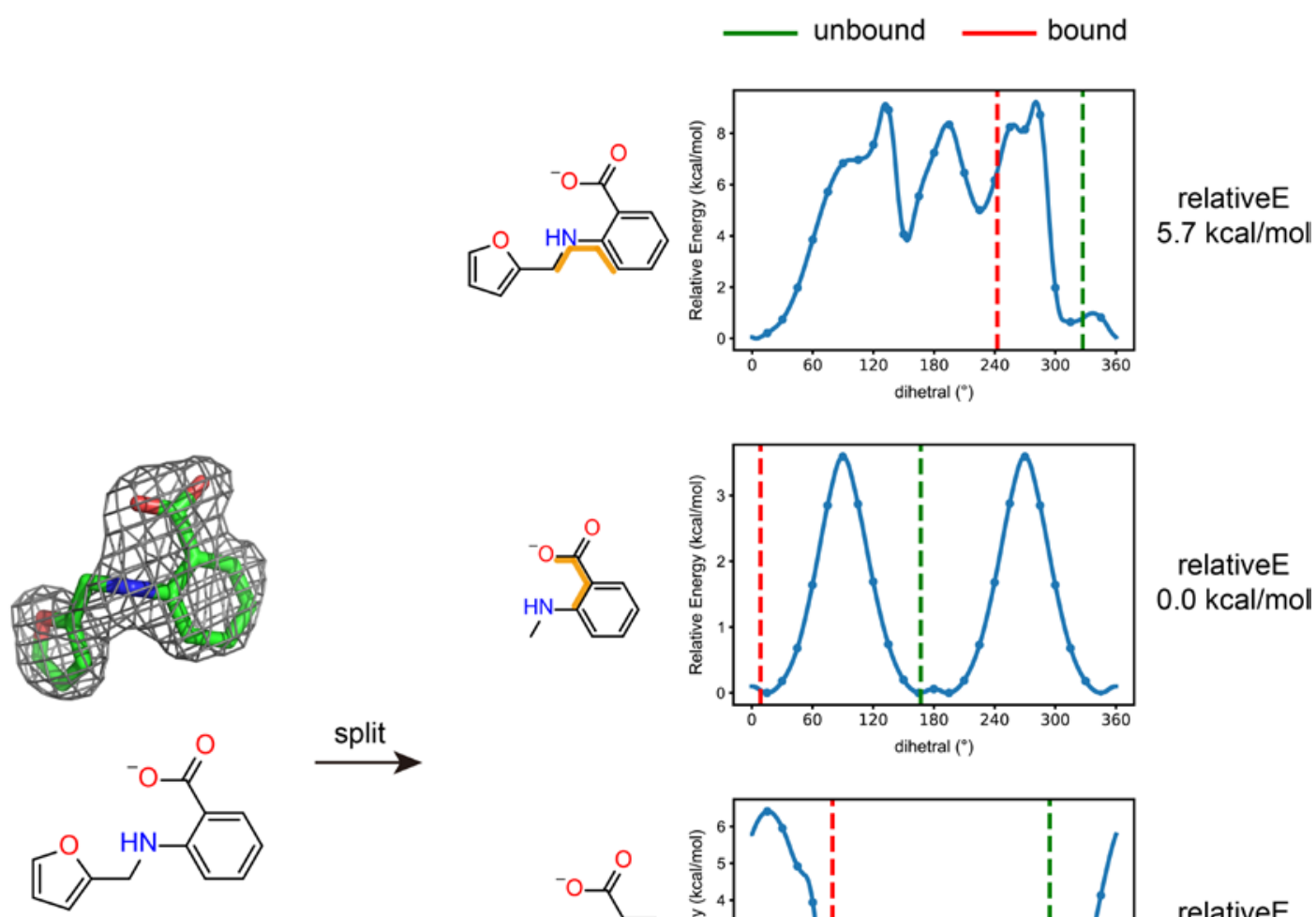

PDB: 3RF5 Ligand: FUZ

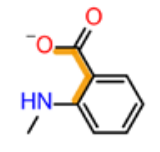

relativeE

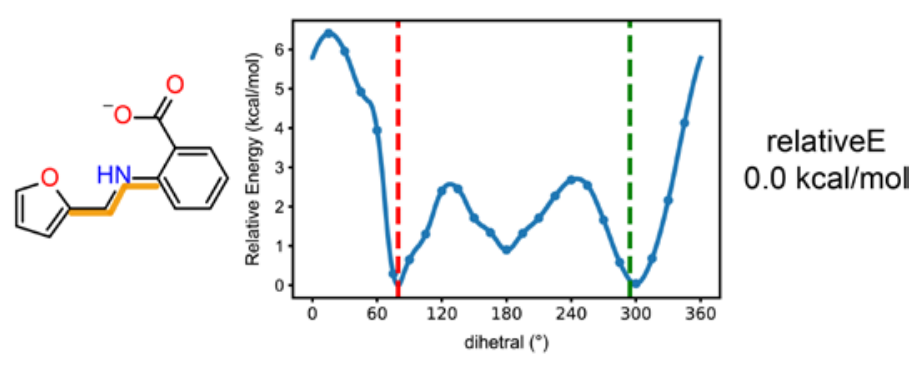

relativeE

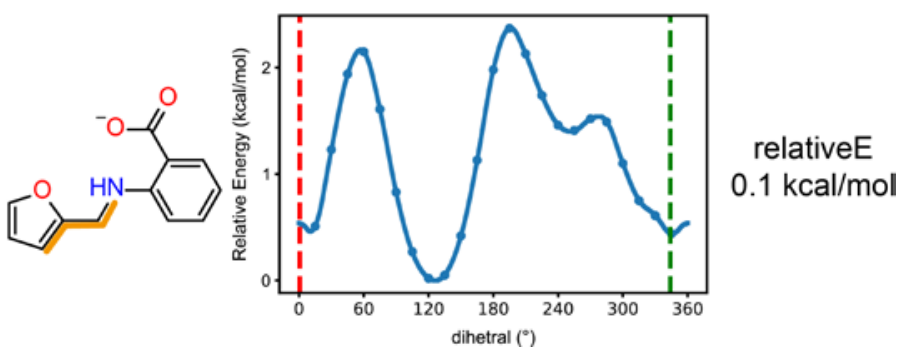

Figure S12. Details of torsion strain energy calculation for the example shown in

Figure 4d. Bound conformation with electron density map and 2D structure of ligand is shown on the left. The split fragments for the torsion scan are shown down the middle, for which the four atoms forming the dihedral angle are marked in purple. Torsion strain energy profiles are shown on the right, as long as the current dihedral angles and torsion energies of ligand bound conformation. 


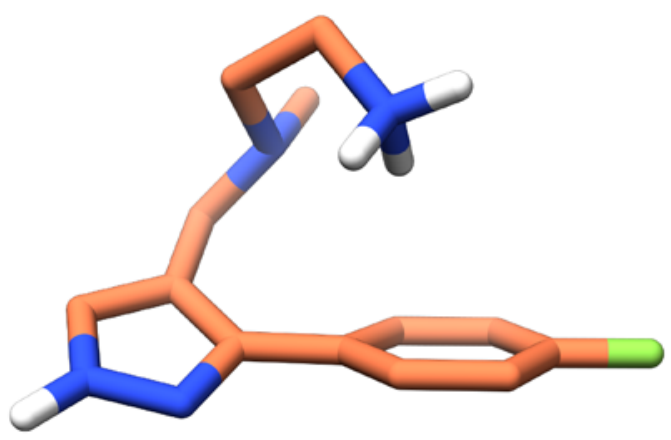

whole ligand conformation

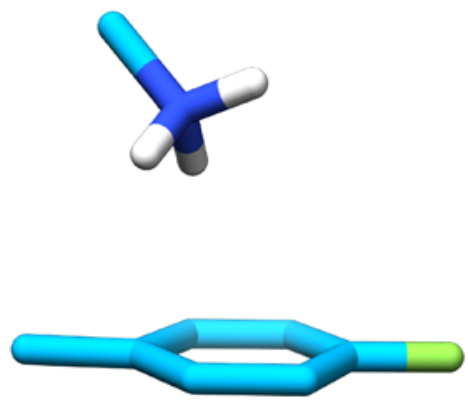

simplified model for intramolecular interaction calculation

Figure S13. Simplified models showing relevant core functional groups of the intramolecular interaction calculation for the example shown in Figure 4e. 

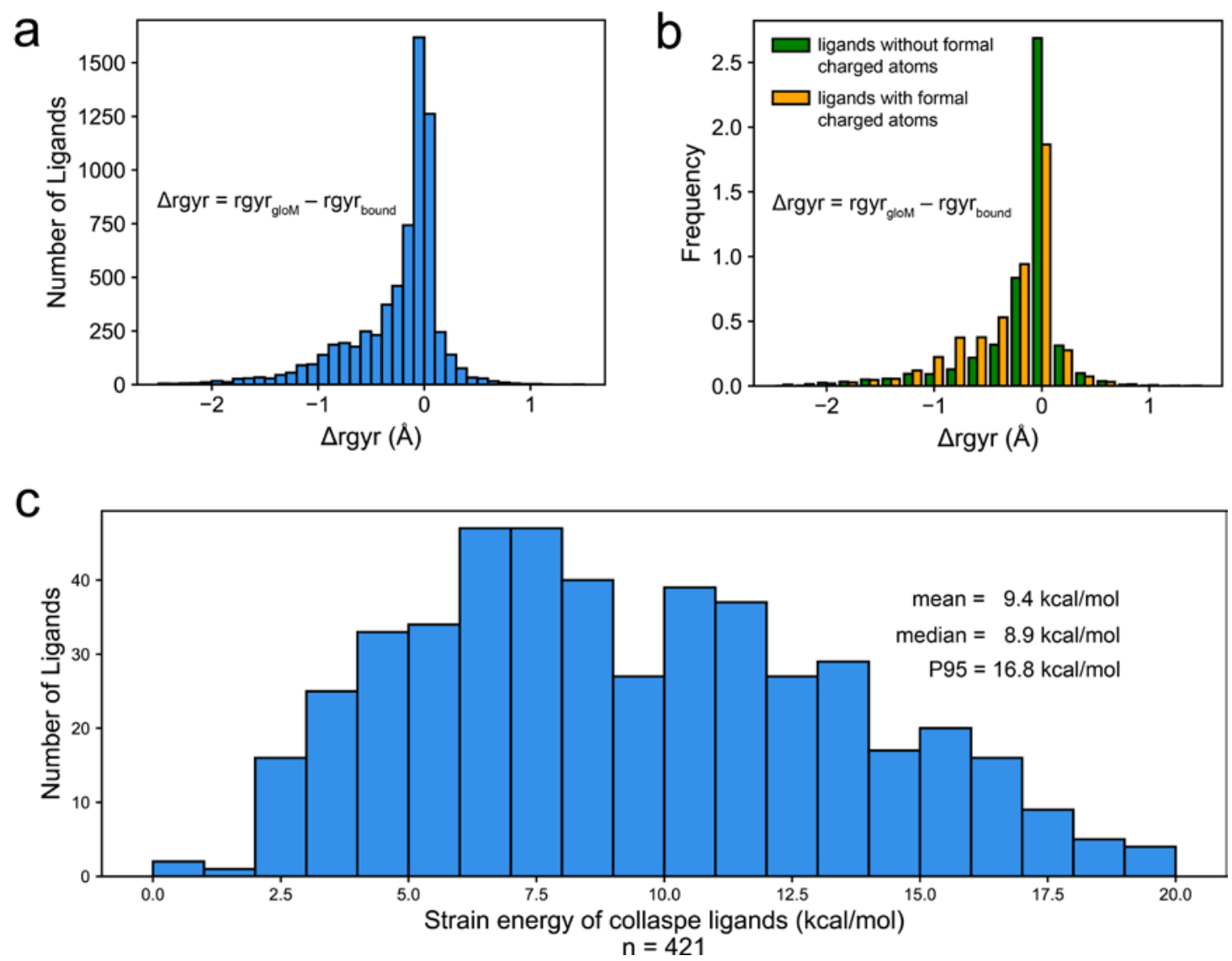

Figure S14. Comparison of rgyr between bound and unbound conformations and strain energy distribution of ligands with large rgyr differences. (a) Distribution of rgyr difference (rgyrglom - rgyr bound) of all successfully calculated cases. (b) Distribution of rgyr difference ( $\operatorname{rgyr}_{\text {glom }}-$ rgyr $\left._{\text {bound }}\right)$ for ligands with and without formal charged atoms. (c) Strain energy distribution of ligands with large rgyr difference $(\Delta \operatorname{rgyr} \leq-1)$. 


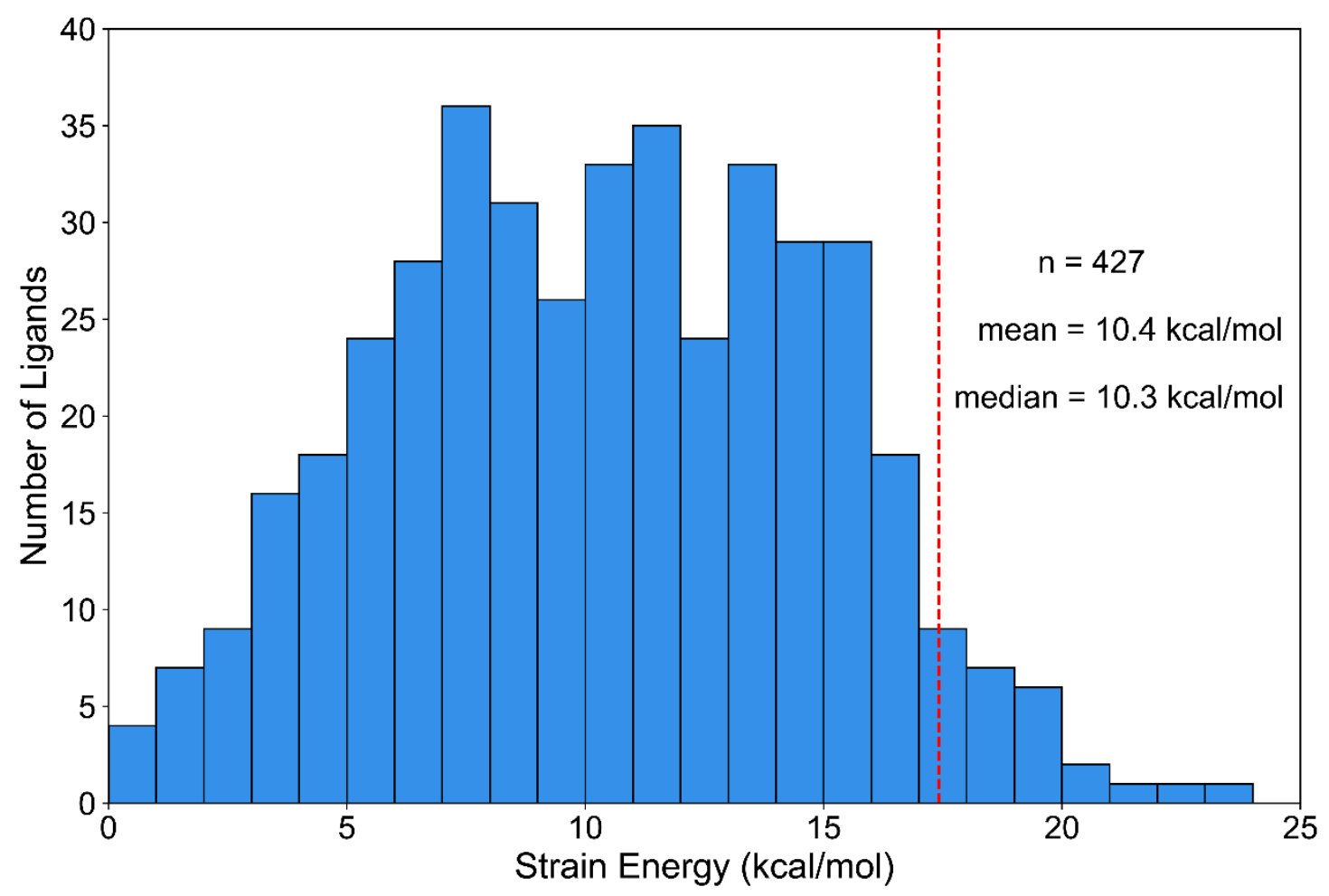

Figure S15. LCSE distribution of 427 ligands with non-local intramolecular interaction(s). The mean/median/P95 is significantly higher than that of the whole dataset. 


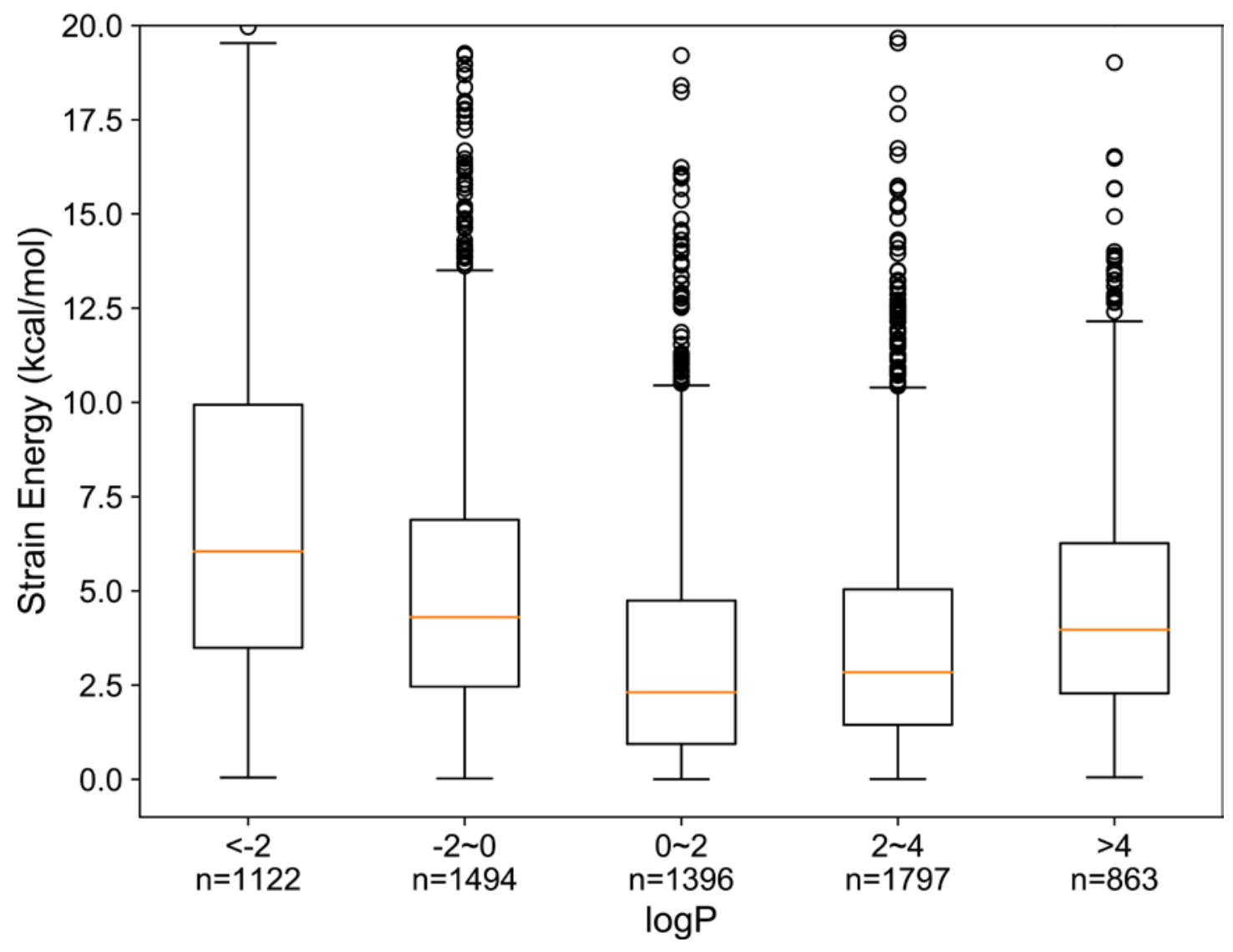

Figure S16. Correlations between LCSE and $\log P$ of ligands. Box-and-whisker plots marking the quartiles and median (orange line) of the distribution of LCSE for logP of ligands. The number of data points $(\mathrm{N})$ in each boxplot is provided with the $\mathrm{x}$-axis labels. 


\section{REFERENCES}

(62) Zhang, X.; He, Y.; Liu, S.; Yu, Z.; Jiang, Z.-X.; Yang, Z.; Dong, Y.; Nabinger, S. C.; Wu, L.; Gunawan, A. M.; Wang, L.; Chan, R. J.; Zhang, Z.-Y., Salicylic Acid Based Small Molecule Inhibitor for the Oncogenic Src Homology-2 Domain Containing Protein Tyrosine Phosphatase-2 (SHP2). J. Med. Chem. 2010, 53, 2482-2493.

(63) Xia, L.; Ruppert, M.; Wang, M.; Panjikar, S.; Lin, H.; Rajendran, C.; Barleben, L.; Stöckigt, J., Structures of Alkaloid Biosynthetic Glucosidases Decode Substrate Specificity. ACS Chem. Biol. 2012, 7, 226-234. 